\title{
ON PRODUCTS OF VECTOR MEASURES
}

\author{
U. K. BANDYOPADHYAY \\ (Received 18 July 1972, revised 9 October 1973) \\ Communicated by E. Strzelecki
}

\section{Introduction}

Products of positive measures play a very important role in analysis. The purpose of this paper is to construct a theory of products of two measures taking values in two (possibly different) Banach spaces. A Fubini theorem is obtained which generalizes the Fubini theorem for the Bochner integral (Dunford and Schwartz (1958), Theorem 9, page 190), and hence also the classical result.

We use the theory of vector integration presented in Dinculeanu (1967). Our arguments rely upon a standard sort of application of the dominated convergence theorem (cf. Dunford and Schwartz (1958), Theorem 9, page 190), and therefore do not appear to generalize to any theory of integration where this theorem is lacking (e.g. Bartle (1956)).

For a Fubini theorem generalized in a different direction see Bogdanowicz (1965). Also see Duchoň (1967) and Duchoň and Kluvanek (1967).

This work originated from my Ph.D. thesis at Carnegie-Mellon University, Pittsburgh. I would like to thank my thesis advisor Professor V. J. Mizel for his help.

\section{Preliminaries}

The scalar field is either real or complex. A $\delta$-ring is a ring of sets closed under countable intersections. A measure space is an ordered 4-tuple $(X, S, U, m)$, where $X$ is a nonempty set, $S$ a $\delta$-ring of subsets of $X, U$ a Banach space, and $m$ a $U$ valued measure on $S$ such that the restriction of its variation to $S$ is finite. This restriction is denoted by $|m|$. (We allow $m$ to be unbounded and its variation to be infinite on $X$ ).

Consider two Banach spaces $V, W$ and a continuous bilinear mapping $(u, v) \rightarrow u v$ of $U \times V$ into $W$ with norm 1 (i.e. $\|u v\| \leqq\|u\|\|v\|)$. The following lemma is used in the next section.

Lemma 1. An m-measurable function $h: X \rightarrow V$ is m-integrable if and only if the function $\|h\|$ is $|m|$-integrable. And in this case we have 


$$
\left\|\int_{X} h d m\right\| \leqq \int_{X}\|h\| d|m|
$$

(See Dinculeanu (1967); Proposition 4, page 122; line 3, page 137.)

\section{Product measures}

We consider first two measure spaces $(X, S, U, m)$ and $(Y, T, V, n)$, a Banach space $W$, and a continuous bilinear mapping (with norm 1) $\Phi:(u, v) \rightarrow u v$ of $U \times V$ into $W$.

Let $S \times T$ denote the $\delta$-ring generated by the measurable rectangles $A \times B(A \in S, B \in T)$. For any $y \in Y$, the $Y$-section $C_{y}$ of a set $C$ in $S \times T$ is a set in $S$ defined by

$$
C_{y}=\{x \in X:(x, y) \in C\} .
$$

The following theorem leads to the definition of a product measure.

THEOREM 1. (i) For each given set $C$ in $S \times T$, the $U$-valued function $y \rightarrow m\left(C_{y}\right)$ on $Y$ is n-integrable.

(ii) The mapping $m \times n$ of $S \times T$ into $W$, defined by

is a measure.

$$
(m \times n)(C)=\int_{Y} m\left(C_{y}\right) d n(y) \quad(C \in S \times T),
$$

(iii) $m \times n$ is the only $W$-valued measure on $S \times T$ for which

$$
(m \times n)(A \times B)=m(A) n(B) \equiv \Phi(m(A), n(B)) \quad(A \in S, B \in T) .
$$

(iv) $|m \times n| \leqq|m| \times|n|$.

(v) $|m \times n|=|m| \times|n|$,

if $\|u v\|=\|u\|\|v\|(u \in U, v \in V)$.

Proof. (i) Let $Q$ denote the collection of all sets $C$ in $S \times T$ such that the function $y \rightarrow m\left(C_{y}\right)$ is $n$-integrable. $Q$ Clearly contains the ring $Q_{0}$ generated by the measurable rectangles, every member of $Q_{0}$ being a finite disjoint union of such rectangles. Also, the monotone class with respect to $S \times T$, generated by $Q_{0}$, is precisely the $\delta$-ring $S \times T$ [Dinculeanu (1967), Proposition 16, page 11]. Hence it is sufficient to show that $Q$ is a monotone class with respect to $S \times T$, i.e. $C=\lim C^{k} \in Q$ for every monotone sequence $\left\{C^{k}\right\}$ in $Q$ such that $C \in S \times T$.

For any fixed $y \in Y,\left\{C_{y}^{k}\right\}$ is a montone sequence of sets in $S$ such that $\lim C_{y}^{k}=C_{y}$. And $\bigcup_{k} C^{k}$ is contained in a member of $Q_{0}$ which in turn is contained in some measurable rectangle, say $A \times B$ [Dinculeanu (1967), Corollary to Proposition 10, page 6].

Hence

and

$$
m\left(C_{y}^{k}\right) \rightarrow m\left(C_{y}\right) \quad(y \in Y) \quad \text { [Dinculeanu (1967), page 18] }
$$




$$
\left\|m\left(C_{y}^{k}\right)\right\| \leqq|m|(A) \chi_{B}(y) \quad(y \in Y ; k=1,2, \cdots) .
$$

It follows now from the dominated convergence theorem [Dinculeanu (1967), Theorem 3, page 136] that $C \in Q$.

The proof of (ii) is now clear and (iii) follows from a standard result [Dinculeanu (1967), Proposition 6, page 24].

(iv) We need only observe that for every $C \in S \times T$ and every finite partition $\left\{C^{k}\right\}$ of $C$ into sets of $S \times T$, we have

$$
\begin{aligned}
\Sigma\left\|(m \times n)\left(C^{k}\right)\right\| & =\Sigma\left\|\int_{Y} m\left(C_{y}^{k}\right) d n(y)\right\| \\
& \leqq \Sigma \int_{Y}|m|\left(C_{y}^{k}\right) d|n|(y) \\
& =\Sigma(|m| \times|n|)\left(C^{k}\right) \\
& =(|m| \times|n|)(C) .
\end{aligned}
$$

(v) By virtue of (iii) and (iv), (v) would follow if we show that

$$
|m \times n|(A \times B) \geqq|m|(A)|n|(B)
$$

for an arbitrary measurable rectangle $A \times B$. If $\left\{A_{i}: i=1, \cdots, I\right\}$ and $\left\{B_{j}: j=1, \cdots, J\right\}$ are finite partitions of $A$ and $B$ into sets of $S$ and $T$ respectively, then $\left\{A_{i} \times B_{j}: i=1, \cdots, I ; j=1, \cdots, J\right\}$ is a finite partition of $A \times B$ into sets of $S \times T$, and we have

$$
\begin{aligned}
|m \times n|(A \times B) & \geqq \sum_{i, j}\left\|(m \times n)\left(A_{i} \times B_{j}\right)\right\| \\
& =\sum_{i, j}\left\|m\left(A_{i}\right) n(B)_{j}\right\| \\
& =\sum_{i, j}\left\|m\left(A_{i}\right)\right\|\left\|n\left(B_{j}\right)\right\| \\
& =\left(\sum_{i}\left\|m\left(A_{i}\right)\right\|\right)\left(\sum_{j}\left\|n\left(B_{j}\right)\right\|\right)
\end{aligned}
$$

This implies (*) since the partitions of $A$ and $B$ are arbitrary.

Definition. The measure $m \times n$ of Theorem 1 will be called the product of the measures $m$ and $n$ corresponding to the Banach space $W$ and the bilinear mapping $\Phi$.

REMARK. The existence of the product measure can be established by using a standard theorem [Dinculeanu (1967), Theorem 3, page 76] on the extension of vector measures. However, one must show first that the product defined on $Q_{0}$ in the obvious way is indeed a measure whose variation (restricted to $Q_{0}$ ) is finite.

For the following Fubini theorem we consider three more Banach spaces $E, F, G$ and three continuous bilinear mappings (with norm 1) 


$$
\begin{aligned}
& (e, w) \rightarrow e w \text { of } E \times W \text { into } G, \\
& (e, u) \rightarrow e u \text { of } E \times U \text { into } F, \\
& (f, v) \rightarrow f v \text { of } F \times V \text { into } G,
\end{aligned}
$$

such that

$$
e(u v)=(e u) v \quad(e \in E, u \in U, v \in V) .
$$

THEOREM 2. Let $h: X \times Y \rightarrow E$ be an $(|m| \times|n|)$-integrable function.

(i) The E-valued function $x \rightarrow h(x, y)$ on $X$ is $m$-integrable for $|n|$-almost all fixed $y \in Y$.

(ii) The F-valued function $y \rightarrow \int_{X} h(x, y) d m(x)$, defined $|n|-a . e$. on $Y$, is n-integrable.

(iii) $\int_{X \times Y} h d(m \times n)=\int_{Y}\left[\int_{X} h(x, y) d m(x)\right] d n(y)$.

ProOF. (i) and (ii) are easily obtained by an application of the Fubini theorem for positive measures to the $(|m| \times|n|)$-integrable function $\|h\|$ on $X \times Y$. (See Lemma 1.)

To prove (iii) we first assume that

Then

$$
h=e \chi_{c} \quad(e \in E, C \in S \times T) .
$$

Hence

$$
h(x, y)=e \chi_{c_{y}}(x)
$$

$$
\begin{aligned}
\int_{Y}\left[\int_{X} h(x, y) d m(x)\right] d n(y) & =\int_{Y}\left[\operatorname{em}\left(C_{y}\right)\right] d n(y) \\
& =e\left[\int_{Y} m\left(C_{y}\right) d n(y)\right] \\
& =e(m \times n)(C) \\
& =\int_{X \times Y} h d(m \times n)
\end{aligned}
$$

by [Dinculeanu (1967), A routine extn. of Proposition 6, page 123].

It is clear now that (iii) holds for all simple functions. For an arbitrary $(|m| \times|n|)$-integrable function $h$, there exists a sequence $\left\{h_{k}\right\}$ of simple functions such that

and

$$
h_{k} \rightarrow h
$$

$$
\left\|h_{k}\right\| \leqq\|h\|,(|m| \times|n|) \text {-a.e. }
$$

(See [Dinculeanu (1967), Theorem 2, page 99].)

For $|n|$-almost all fixed $y \in Y$, 
and

$$
h_{k}(x, y) \rightarrow h(x, y)
$$

$$
\left\|h_{k}(x, y)\right\| \leqq\|h(x, y)\|,|m| \text {-a.e. }
$$

Hence, by the dominated convergence thorem [Dinculeanu (1967), Theorem 3, page 136],

$$
\int_{X} h_{k}(x, y) d m(x) \rightarrow \int_{X} h(x, y) d m(x) \quad|n| \text {-a.e. on } Y .
$$

Since (by Lemma 1),

$$
\left\|\int_{X} h_{k}(x, y) d m(x)\left|\leqq \int_{X}\|h(x, y)\| d\right| m|(x) \quad| n \mid\right. \text {-a.e. }
$$

another application of the same theorem yields that

$$
\int_{Y}\left[\int_{X} h_{k}(x, y) d m(x)\right] d n(y) \rightarrow \int_{Y}\left[\int_{X} h(x, y) d m(x)\right] d n(y) .
$$

This implies (iii), because

$$
\int_{X \times Y} h_{k} d(m \times n) \rightarrow \int_{X \times Y} h d(m \times n)
$$

and because we have

$$
\int_{Y}\left[\int_{X} h_{k}(x, y) d m(x)\right] d n(y)=\int_{X \times \mathbf{Y}} h_{k} d(m \times n)
$$

for all $k$.

REMARKS. If $m$ and $n$ are scalar valued and if $\Phi$ denotes the regular product of numbers, then $|m \times n|=|m| \times|n|[$ Theorem $1(v)]$ and Theorem 2 reduces to the standard Fubini theorem for $(m \times n)$-integrable functions. However, as the following example shows, $|m \times n|$-integrability is not sufficient in the general case. Let $X=Y=(0,1), S=T=$ the Lebesgue measurable sets of $(0,1)$, $U=V=R^{2}, W=R, \Phi$ the standard inner product in $R^{2}, m(A)=\mu(A)\langle 1,0\rangle$, and $n(B)=\mu(B)\langle 0,1\rangle$, where $\mu$ is the Lebesgue measure on $(0,1) . m \times n$ is then the zero measure on $S \times T$. The function $h:(x, y) \rightarrow 1 / x$ on $X \times Y$ is $|m \times n|$-integrable but fails to satisfy (i) of Theorem 2 .

\section{References}

R. G. Bartle (1956), 'A general bilinear vector integral', Studia Math. 15, 337-352.

W. M. Bogdanowicz (1965), 'Fubini theorems for generalized Lebesgue-Bochner-Stieltjes integral', Proc. Japan Acad. 41, 979-983.

N. Dinculeanu (1967), Vector measures, (Pergamon, New York, 1967.) 
M. Duchon (1967), 'On the projective tensor product of vector valued measures', Mat. Casopis Sloven. Akad. Vied 17, 113-120.

M. Duchoň and I. Kluvánek (1967), 'Inductive tensor product of vector valued measures', Mat. Časopis Sloven. Akad. Vied 17, 108-112.

N. Dunford and J. T. Schwartz (1958), Linear operators. I: General theory, Pure and Appl. Math. 7, (Interscience, New York, 1958).

\section{Department of Mathematics}

University of Cincinnati

Cincinnati, Ohio 45221

U. S. A. 